*This manuscript should be cited as:

Zabelina, D. L., Clay, J. Z., \& Upshaw, J. D. (in press). Imagination, anxiety, and loneliness during the COVID-19 pandemic. Creativity Research Journal.

\title{
Imagination, Anxiety, and Loneliness During the COVID-19 Pandemic
}

\author{
Darya L. Zabelina, John Z. Clay, \& Joshua D. Upshaw \\ University of Arkansas \\ Psychological Science Department \\ 480 Campus Drive \\ Fayetteville, AR 72701 \\ USA
}

Author Note

Correspondence concerning this article should be addressed to Darya L. Zabelina, Department of Psychological Science, 480 Campus Drive, Fayetteville, AR 72701. Ph: 479-5755813. Email: dlzabeli@uark.edu. ORCID iD https://orcid.org/0000-0002-0313-7358 


\begin{abstract}
Imagination is relevant in many aspects of our lives, and has been associated with creativity and overall cognitive development, yet imagination may also have a dark side. In two studies we examined the link between imagination, anxiety, and loneliness during the COVID-19 pandemic. US college students $(N=101$, Study 1$)$ and participants from an international community sample $(N=61$, Study 2$)$ were tested around the time that rates of COVID-19 cases were escalating. Across both samples, we found that spending more time in one's imagination was associated with elevated levels of anxiety. Furthermore, frequency of imagination interacted with loneliness in predicting changes in anxiety during, compared to before the pandemic. Specifically, lonely people who spent more time imagining experienced the largest increases in anxiety during, compared to before the pandemic. In Study 1 valence of imagination and changes in anxiety were also moderated by feelings of loneliness, although this effect was not replicated in Study 2 . Results indicate that at least some features of imagination, particularly how much time people spend in imaginative states, may be associated with negative mood states, such as feelings of anxiety. Implications and future directions are discussed.
\end{abstract}

Keywords: Imagination, anxiety, loneliness, COVID-19 


\section{Imagination, Anxiety, and Loneliness During the COVID-19 Pandemic}

On March $11^{\text {th }}, 2020$, the World Health Organization declared the coronavirus (COVID19) outbreak a pandemic, later suggesting that all individuals, regardless of whether or not they had come into contact with the virus, practice self-quarantine and various safety measures until the situation improves (World Health Organization, 2020). Though these practices have shown to be effective in slowing the spread of the virus and preserving individual physical health, they may also come at a costs. Indeed, the U.S. Census Bureau reported that a third of Americans now show signs of clinical anxiety amid the COVID-19 pandemic (Centers for Disease Control and Prevention, 2020). Research further shows that in China, where the outbreak was first reported, people who spent more time focusing on the COVID-19 had higher generalized-anxiety symptoms (Huang \& Zhao, 2020). Other studies found that forced quarantines can result in feelings of detachment from others, irritability, distress, post-traumatic stress, and anxiety (Bai et al., 2004; Sprang \& Silman, 2013; Taylor et al., 2008).

Forced social isolation is also likely to result in feelings of loneliness (Steptoe et al., 2013 ) - i.e., a discrepancy in perception between one's existing social relationships and the social relationships one wishes to have (Peplau, 1982). A number of studies have linked loneliness with lower life satisfaction (Schumaker et al., 1993) and mental health issues such as anxiety and psychosis (Sündermann et al., 2014). The relationship between loneliness and anxiety appears to be bi-directional. For example, anxiety has been shown to be linked with greater loneliness among clinical samples, specifically among people with social anxiety disorder (Eres et al., 2020). Other research suggests that loneliness is more likely to be a significant risk factor for 
anxiety and depression symptoms (Hoffart et al., 2020; Palgi et al., 2020), and is a powerful predictor of death anxiety (Çakar, 2020). Additionally, tactics that relieve loneliness have been shown to have an alleviating effect on anxiety (Cauberghe et al., 2020).

Feelings of loneliness have also been associated with an increased frequency of imaginative thinking - i.e., formation of new ideas, images, or concepts in one's mind's eye (Zabelina, in press). Previous literature shows that lonely children tend to develop complex fantasy lives and spend a great deal of time living them out (Rhue \& Lynn, 1987). Loneliness has also been associated with daydreaming - a construct closely related to imagination (Poerio et al., 2015; Poerio et al., 2016), particularly when daydreaming is focused on people who are not close to oneself (Mar et al., 2012).

Rich fantasy and imagination are generally considered positive states, often resulting in creative behaviors and innovation (LeBoutillier \& Marks, 2003; Samli, 2011). Literature on children's cognitive development shows that securely attached children are more likely to engage in imaginative play and exploration, in turn facilitating their creative thinking (Holmes, 2015). Mental imagery and visual representations have also been shown to be linked with improved reading and mathematics skills (Arcavi, 2003; Sadoski et al., 1990). And frequentt engagement in imagination during childhood is related to a higher social understanding (Taylor et al., 2004). Interestingly, threatening situations have also been found to evoke more imaginative and creative responses, suggesting imagination may serve a survival purpose (Riley \& Gabora, 2013).

Imagination might also have a dark side. For instance, frequent imagination has been linked with higher levels of anxiety and impulsivity (Zabelina \& Condon, in press), while fantasy proneness has been associated with higher levels of emotional distress, maladaptive coping strat- 
egies, and higher likelihood of rumination and catastrophizing (Bacon \& Charlesford, 2018). Rumination may especially share common cognitive mechanisms of frequent imagination, as a recent neuroimaging meta-analysis found a positive association between rumination and the default mode network (Zhou et al., 2020) - a set of neural regions known to be active implicated in imaginative thinking (Beaty et al., 2018).

Although previous work provides some indication that imagination and anxiety may be linked, it is not yet known which features of imagination are associate with anxiety states. Furthermore, the nature of the association between imagination and anxiety is yet to be investigated in the time of global pandemic. Although literature tends to focus on the detail with which people are able to imagine (e.g., Brown et al., 2002), a recent model of imagination proposed that imagination consists of at least four distinct components; (1) frequency - the amount of time one spends in imaginative states, (2) complexity of imagination - the detail with which one imagines, (3) emotional valence - the degree to which one typically imagines in a positive or negative manner, and (4) directedness - how goal-oriented (vs. "free-floating") one's imagination tends to be (Zabelina \& Condon, in press). In the present study we used this model to make predictions about anxiety and feature of imagination, further examining the moderating role of loneliness. We expected that frequency, but not other features of imagination would be associated with higher anxiety both before and during COVID-19 pandemic. This hypothesis is based on previous work showing associations between frequency of imagination and elevated anxiety and lower levels of emotional stability and well-being (Zabelina \& Condon, in press).

Complexity of imagination was not predicted to be associated with individual differences in anxiety. Previous work on the complexity of imagination reported that imagining constructive behaviors in the face of stressful situations is effective in reducing negative emotions (Taylor et 
al., 1998), especially when the imaginative thinking is high in detail (Brown et al., 2002; Jing et al., 2016). In clinical settings, imagining constructive behaviors is a widely used tool for the treatment of anxiety disorders, including obsessive-compulsive disorder and generalized anxiety disorder (Garry \& Polaschek, 2000; Guitard et al., 2019; Stewart et al., 2016). Because this was not an experimental study which manipulated the imagination of constructive behaviors, we expected to see no associations between complexity of imagination and anxiety. Furthermore, because both frequency of imagination and loneliness have previously been associated with elevated anxiety, we hypothesized an interaction between frequency of imagination and loneliness in predicting changes in anxiety during, compared to before the COVID-19 pandemic.

All data were collected online between March 19 - April 29, 2020 in university students in the United States (Study 1), and on March 23, 2020 in an international community sample (Study 2). All participants completed the Four-Factor Imagination Scale (FFIS; Zabelina \& Condon, in press), the Social and Emotional Loneliness Scale (Gierveld \& Van Tilburg, 2006), and the PROMIS Anxiety measure (Cella et al., 2010), reporting on their levels of anxiety before and during the pandemic (i.e., in the past week). The dependent variable was the change (the difference score) in anxiety levels during vs. before the pandemic.

\section{Study 1}

\section{Method}

\section{Participants}

One-hundred-and-one university students (44 male, 56 female, 1 unreported), mean age = 19.77, $S D=1.87, \min =17, \max =28$ participated in the current study. Four participants were American Indian, Native American, or Alaska Native, 6 Asian or Asian American, 4 Black, African American, or African, 9 Latino or Latina, 1 Middle Eastern or Arab, 1 Native Hawaiian or 
Other Pacific Islander, 73 White or Caucasian, 2 multi-racial, and 1 chose not to disclose. This was a convenience sample, and all interested participants were invited to participate (there were no exclusion criteria). Sensitivity power analysis indicated that for a target power of .90 with our sample size $(N=101)$ and alpha $=.05$, critical $t$-value was $1.98\left(f^{2}=.11 ; \mathrm{G} *\right.$ Power; Erdfelder et al., 1996). Twelve participants reported that they had been diagnosed with a psychiatric or psychological disorder (2 with anxiety, 3 with depression, 6 with anxiety and depression, and one “other").

Fifteen participants indicated that they personally knew at least one person who had been diagnosed with COVID-19, and three participants said they personally knew someone who died from COVID-19. All participants completed the consent form prior to participation and were compensated with class credit. The study was approved by the local Institutional Review Board (IRB).

\section{Experimental Design and Procedure}

The study was correlational in nature. Participants completed all questionnaires online between March 19 - April 29, 2020. Questionnaires included FFIS (Zabelina \& Condon, in press), the Patient-Reported Outcomes Measurement Information System (PROMIS, Cella et al., 2010), and the Social and Emotional Loneliness Scale (Gierveld \& Van Tilburg, 2006), demographics, and other questionnaires as part of a larger study. There were two attention check questions which asked participants to respond to a question "Select somewhat agree (or disa-

gree) to continue." If participants answered the first question incorrectly $(n=2)$, they received a warning that if they miss future attention checks, their data will not be usable. If they answered the second attention check question incorrectly, they were removed from analysis $(n=0)$. 


\section{Measures}

The Four-Factor Imagination Scales (FFIS; Zabelina \& Condon, in press) is a 26-item scale that measures four factors of imagination on a 6-item Likert-type scale, from 1 = very inaccurate to $6=$ very accurate. The four factors include frequency - the amount of time one spends in imaginative states (e.g., "I find myself lost in imagination very frequently"), complexity - the detail with which one imagines (e.g., "My fantasies are less detailed than most people's"), emotional valence - the degree to which one's imagination tends to be positively or negatively valenced (e.g., "I visualize negative outcomes for the future of the world"), and directedness - a measure of how goal-oriented (vs. "free-floating") one's imagination tends to be (e.g., "My daydreams are directed towards a specific outcome"). Participants were asked to indicate how accurately each statement described them. The FFIS has good internal consistency of $\alpha=.87(\alpha=.76$ - .93 for the subscales), and both convergent and discriminant validity (Zabelina \& Condon, in press).

The Patient-Reported Outcomes Measurement Information System (PROMIS; Cella et al., 2010) is a four-item measure that assesses anxiety levels of a 4-item Likert-type scale from $1=$ almost never to 4 = almost always. Example of the items are "I found it hard to focus on anything other than my anxiety" and "My worries overwhelmed me." Participants filled out the PROMIS twice - once indicating their anxiety levels prior to COVID-19, and once reporting on their anxiety levels "over the past week." Change in anxiety levels was calculated as the difference score for anxiety levels during minus anxiety level before COVID-19. The PROMIS developers report the scale's reliability at $\alpha=.89$, and good construct validity (Cella et al., 2010). The scale has demonstrated good convergent validity with legacy anxiety measures (Kroenke et al., 2014). 
The Emotional and Social Loneliness Scale (Gierveld \& Van Tilburg, 2006) consists of six items on which participants indicated their feelings of emotional and social loneliness at the time of the experiment (i.e., reporting on their state loneliness) on a Likert-type scale from $1=$ no! to 5 = yes!. Examples of statements include, "I miss having people around" and "I often feel rejected." The Emotional and Social Loneliness Scale has acceptable reliability, $\alpha=.76$, and congruent validity (Gierveld \& Van Tilburg, 2006).

Questions relating to COVID-19. We asked participants a series of questions regarding their personal experience with COVID-19. Namely, we asked if they personally knew anyone who was diagnosed with COVID -19, and whether they personally knew anyone who has died from COVID-19. Due to the program error, data for these questions for the first 15 participants were not collected, thus $n=86$ for questions pertaining to COVID-19.

\section{Results}

Descriptive statistics and zero-order correlations are depicted in Table 1. As predicted, higher frequency of imagination was significantly associated with higher levels of anxiety during COVID-19 $(r=.27, p=.006)$. Frequency of imagination was not significantly associated with anxiety prior to COVID-19 $(r=.15, p=.135)$. Frequency of imagination was also significantly correlated with higher levels of loneliness $(r=.32, p=.001)$. More positive imagination was linked with lower levels of anxiety both before $(r=-.31, p=.002)$ and during COVID-19 $(r=-$ $.43, p<.001)$, and with reduced feelings of loneliness $(r=-.55, p<.001)$. Older people reported more goal-directed imagination $(r=.22, p=.027)$, and higher levels of loneliness $(r=.20, p=$ $.043)$. 


\section{Table 1}

Pearson Correlation Coefficients for Imagination, Anxiety, Loneliness, and Age in College Students

\begin{tabular}{|c|c|c|c|c|c|c|c|c|}
\hline & 1 & 2 & 3 & 4 & 5 & 6 & 7 & 8 \\
\hline $\begin{array}{l}\text { 1. FFIS Frequency } \\
\text { 2. FFIS Complexity } \\
\text { 3. FFIS Emotional Valence } \\
\text { 4. FFIS Directedness } \\
\text { 5. Anxiety before COVID-19 } \\
\text { 6. Anxiety during COVID-19 } \\
\text { 7. Loneliness } \\
\text { 8. Age }\end{array}$ & -- & $\begin{array}{l}.20^{*} \\
--\end{array}$ & $\begin{array}{l}-.23 \\
.37 * * * \\
--\end{array}$ & $\begin{array}{l}.21^{*} \\
-.05 \\
.12 \\
--\end{array}$ & $\begin{array}{l}.15 \\
-.06 \\
-.31^{* *} \\
.05 \\
--\end{array}$ & $\begin{array}{l}.27^{* *} \\
-.09 \\
-.43^{* * *} \\
.08 \\
.40^{* * *} \\
--\end{array}$ & $\begin{array}{l}.32^{* *} \\
-.04 \\
-.55^{* * *} \\
-.10 \\
.35^{* * *} \\
.41^{* *} \\
--\end{array}$ & $\begin{array}{l}.13 \\
.09 \\
-.11 \\
.22 * \\
-.08 \\
.11 \\
.20^{*} \\
--\end{array}$ \\
\hline $\begin{array}{l}\text { Mean } \\
\text { SD }\end{array}$ & $\begin{array}{l}3.60 \\
.99\end{array}$ & $\begin{array}{l}4.35 \\
.75\end{array}$ & $\begin{array}{l}4.59 \\
.94\end{array}$ & $\begin{array}{l}3.75 \\
.91\end{array}$ & $\begin{array}{l}1.63 \\
.71\end{array}$ & $\begin{array}{l}1.73 \\
.80\end{array}$ & $\begin{array}{l}2.58 \\
.78\end{array}$ & $\begin{array}{l}19.77 \\
1.87\end{array}$ \\
\hline
\end{tabular}

Note. $N=101 . \mathrm{FFIS}=$ Four Factor Imagination Scale. ${ }^{* *} p<.001, * * p<.01, * p<.05$

We further tested for gender differences in the variables of interest. Independent-sample $t$-tests indicated that women reported higher levels of anxiety before and during COVID-19, but there were no gender differences in imagination or loneliness levels, nor in the changes in anxiety during vs. before COVID-19 pandemic (Table 2). 


\section{Table 2}

Gender Differences in Imagination, Anxiety, and Loneliness in College Students

\begin{tabular}{|c|c|c|c|c|c|c|c|}
\hline & \multicolumn{2}{|c|}{ Male } & \multicolumn{2}{|c|}{ Female } & \multirow[b]{2}{*}{$t$} & \multirow[b]{2}{*}{$p$} & \multirow[b]{2}{*}{$95 \% \mathrm{CI}$} \\
\hline & $\bar{M}$ & $S D$ & $M$ & $S D$ & & & \\
\hline FFIS Frequency & 3.54 & 1.02 & 3.65 & .98 & .54 & .588 & {$[-.51 ; .29]$} \\
\hline FFIS Complexity & 4.28 & .87 & 4.40 & .64 & .83 & .406 & {$[-.43 ; .17]$} \\
\hline FFIS Emotional Valence & 4.53 & .91 & 4.63 & .97 & .56 & .579 & {$[-.48 ; .27]$} \\
\hline FFIS Directedness & 3.56 & .94 & 3.89 & .86 & 1.78 & .078 & {$[-.68 ; .04]$} \\
\hline Anxiety before COVID-19 & 1.47 & .58 & 1.76 & .78 & 2.13 & $.036^{*}$ & {$[-.58 ;-.02]$} \\
\hline Anxiety during COVID-19 & 1.41 & .56 & 1.98 & .87 & 3.74 & $.000 * * *$ & {$[-.87 ;-.27]$} \\
\hline Loneliness & 2.54 & .71 & 2.61 & .85 & .43 & .667 & {$[-.38 ; .25]$} \\
\hline
\end{tabular}

Note. FFIS $=$ Four Factor Imagination Scale. ${ }^{* *} p<.001,{ }^{*} p<.05$

Next, we tested the hypothesis that the frequency of imagination would interact with loneliness in predicting larger increases in anxiety during vs. before the COVID-19 pandemic. A multiple regression analysis was carried out to test this hypothesis. The three predictors explained $7 \%$ of the variance in anxiety change, $\mathrm{R}^{2}=0.07, F(3,96)=2.48, p=.066$. There was no main effect of the frequency of imagination, indicating that frequency of imagination was not generally associated with the change in anxiety levels during vs. before COVID-19. There was also no main effect of loneliness, indicating that loneliness was not linked with the change in anxiety either. As predicted, there was a significant frequency of imagination $\mathrm{x}$ loneliness interaction in predicting change in anxiety levels (Table 3). Estimated means for this interaction were calculated for individuals low (-1 SD) versus high (1 SD) in the frequency of imagination and people low (-1 SD) versus high (1 SD) in loneliness, and are displayed in Figure 1. As can be seen from the figure, lonely people with higher frequency of imagination reported an increase in anxiety levels during vs. before COVID-19. Lonely people who spend less time imagining 
showed a decrease in anxiety levels during vs. before COVID-19. The interaction remained significant controlling for whether or not participants had been diagnosed with psychiatric or psychological disorders, $\beta=.27, t=2.22, p=.029$.

\section{Table 3}

Liner Multiple Regressions Predicting Anxiety Levels During vs. Before COVID-19 in College Students.

$\begin{array}{llllll}\text { B } & \text { SE } & \beta & t & p & 95 \% \mathrm{CI}\end{array}$

DV: Anxiety levels during vs. before COVID-19

$\begin{array}{lllllll}\text { FFIS Frequency } & .12 & .09 & .13 & 1.23 & .222 & {[-.07 ; .28]} \\ \text { Loneliness } & .01 & .09 & .01 & 0.01 & .924 & {[-.17 ; .18]} \\ \text { FFIS Frequency x Loneliness } & .16 & .07 & .23 & 2.30 & .024^{*} & {[.02 ; .29]}\end{array}$

Note. FFIS $=$ Four Factor Imagination Scale. ${ }^{* * *} p<.001,{ }^{*} p<.05$

Figure 1. Anxiety levels during vs. before COVID-19 as a function of frequency of imagination and reported loneliness in college students. More positive values indicate higher levels of anxiety during compared to before COVID-19.

Low Loneliness $\quad \square$ High Loneliness

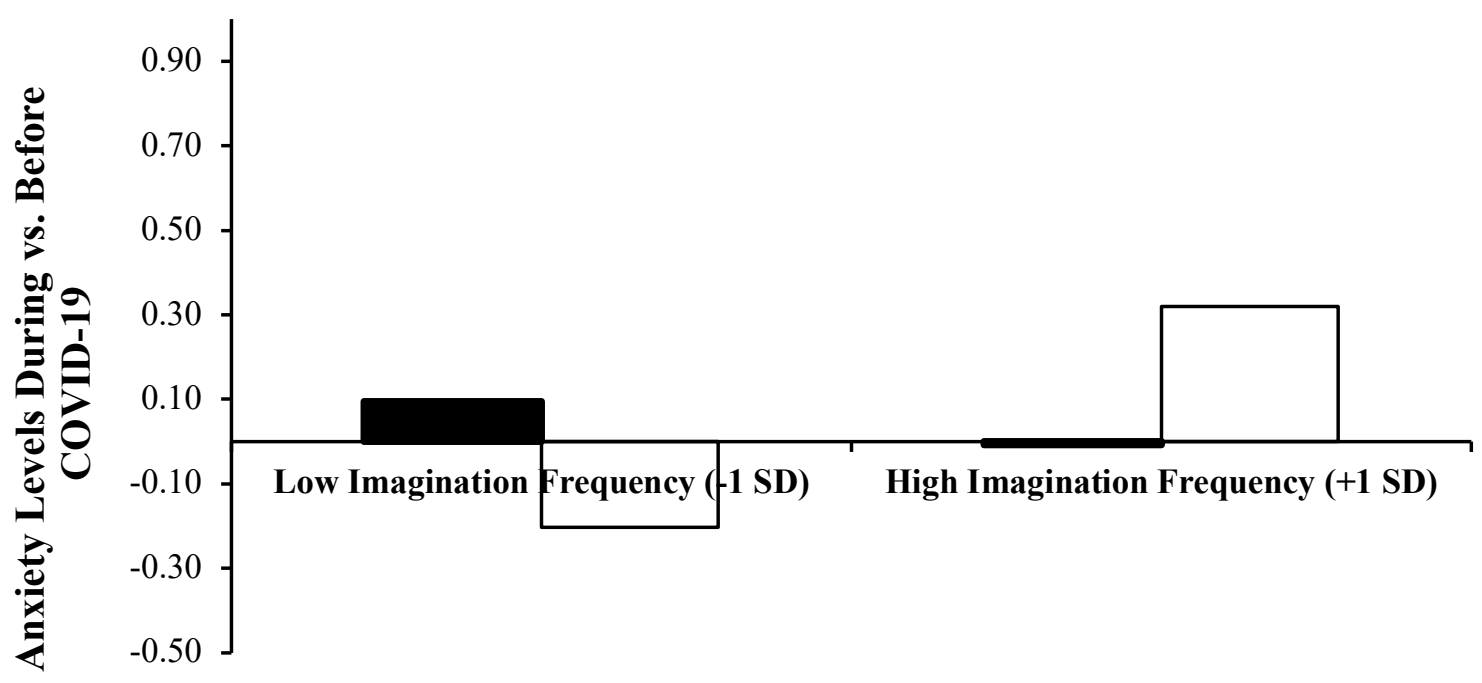


We further performed three separate multiple regression analyses to test the interaction between imagination complexity, emotional valence, and directedness and loneliness in predicting change in anxiety levels during vs. before COVID-19. There was no main effect of complexity of imagination $(\beta=-.04, t=-.36, p=.723)$ or loneliness $(\beta=.09, t=.89, p=.373)$, nor did they interact $(\beta=-.03, t=-.24, p=.811)$ in predicting changes in anxiety levels during vs. before COVID-19. There was also no main effect of the emotional valence of imagination $(\beta=-.07, t=$ $-.54, p=.588)$ or loneliness $(\beta=.02, t=.13, p=.899)$, however their interactive effect was statistically significant $(\beta=-.28, t=-2.73, p=.007)$. Estimated means for this interaction were calculated for individuals with more negative (-1 SD) versus more positive (1 SD) imagination and people low (-1 SD) versus high (1 SD) in loneliness (Figure 2). Namely, lonely people who tend to have more negative imagination showed an increase in anxiety levels during vs. before COVID-19. Interestingly, lonely people who tend to engage in more positive imagination, or people who are not lonely, but report engaging in more negative imagination showed a decrease in anxiety levels during vs. before COVID-19. For the valence of imagination, the three predictors explained $6 \%$ of the variance in anxiety change, $\mathrm{R}^{2}=0.06, F(3,96)=3.27, p=.025$. The interaction remained significant controlling for whether or not participants had been diagnosed with psychiatric or psychological disorder, $\beta=.40, t=4.06, p<.001$. 
Fig 2. Anxiety levels during vs. before COVID-19 as a function of the valence of imagination and reported loneliness in college students. More positive values indicate more anxiety during compared to before COVID-19.

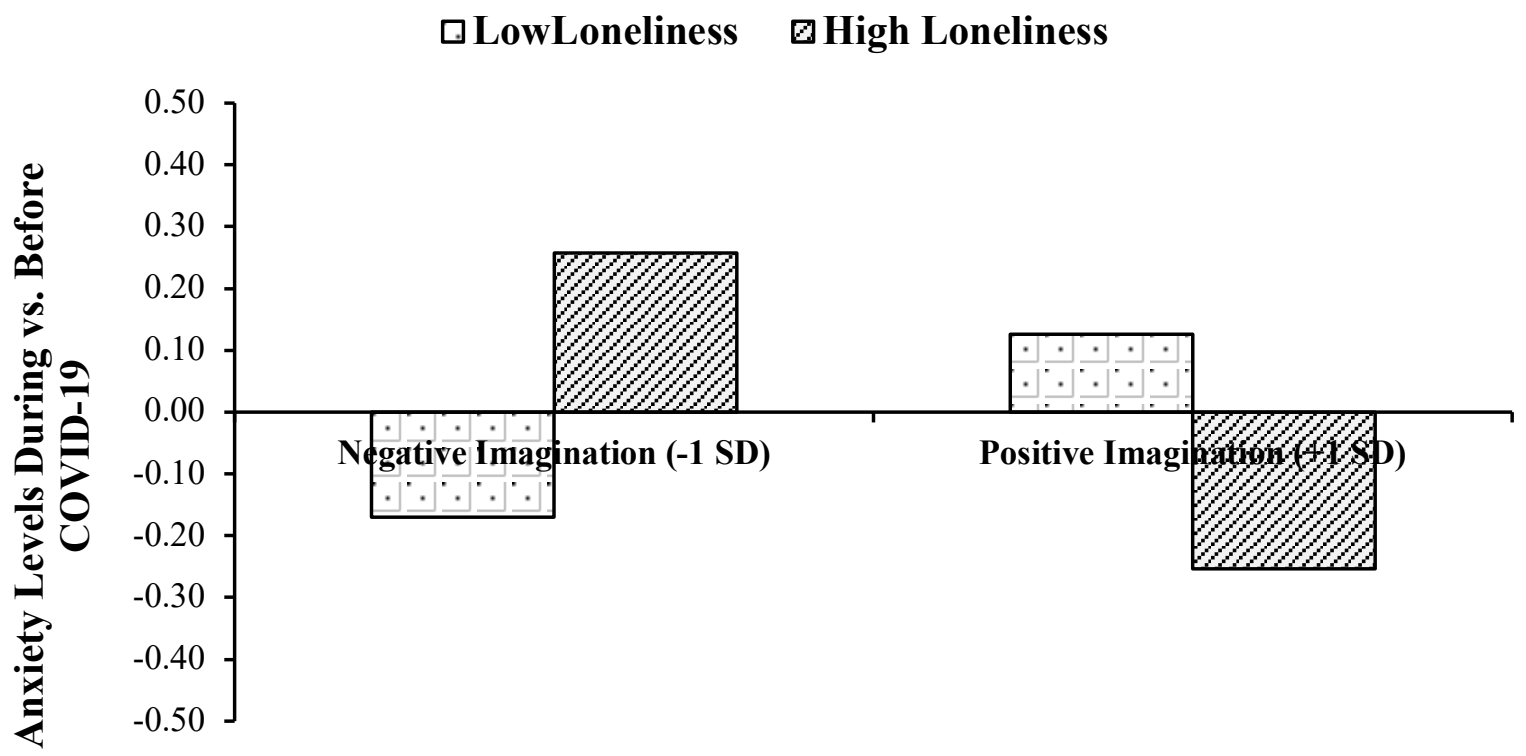

Finally, there was no main effect of directedness of imagination $(\beta=.03, t=.31, p=$ $.758)$ or loneliness $(\beta=.09, t=.86, p=.394)$, nor did they interact $(\beta=-.07, t=-.64, p=.526)$ in predicting the change in anxiety during vs. before COVID-19.

\section{Discussion}

In Study 1 we hypothesized that frequency of imagination would be linked with higher levels of anxiety both before and during COVID-19 pandemic, and that frequency of imagination and loneliness would interact in predicting changes in anxiety during vs. before COVID-19 pandemic. Our hypotheses were partially supported. We found that frequency of imaginative states was associated with higher levels of anxiety levels during (but not before) COVID-19. Furthermore, as predicted, a multiple regression analysis revealed that frequency of imagination and loneliness interacted in predicting elevated anxiety during (vs. before) the pandemic. Previous 
literature reported that frequency of imagination and mind wandering - a construct closely related to imagination, are associated with decreased psychological well-being and elevated anxiety (Killingsworth \& Gilbert, 2010; Mason et al., 2013; Zabelina \& Condon, in press). The results of Study 1 further show that this association remains during the world-wide pandemic, and feelings of loneliness moderates this relationship.

Results also revealed a significant interaction between emotional valence of imagination and loneliness in predicting changes in anxiety. Namely, lonely participants who reported more negative imagination also reported higher levels of anxiety during (vs. before) COVID-19. Interestingly, results also showed that positive imagination or feeling less lonely may also have a protective effect on the elevation of anxiety during the pandemic.

Finally, female (vs. male) participants reported higher levels of anxiety both before and during COVID-19, suggesting that in a college sample, women experience higher anxiety than men regardless of the situation. This result is in line with prior literature showing that women are significantly more likely to develop anxiety disorders compared to men (e.g., McLean et al., 2011).

The goals of Study 2 were twofold. First, we sought to replicate the results of Study 1 in a separate sample. Second, we aimed to examine the generalizability of the results to non-college student populations. Thus, in Study 2 we administered the survey to an international community sample. 


\section{Study 2}

\section{Method}

\section{Participants}

Sixty-one people (29 male, 31 female, 1 unreported), mean age $=33.62, S D=13.78$, $\min$ $=15, \max =72$ participated in the current study. One participant was Asian or Asian American, 7 Black, African American, or African, 2 Latino or Latina, 2 Middle Eastern or Arab, 1 Native Hawaiian or Other Pacific Islander, 47 White or Caucasian, and 1 multi-racial. This was a convenience sample, and all interested participants were invited to participate (there were no exclusion criteria). A sensitivity power analysis indicated that for a target power of .90 , with our sample size $(N=61)$ and alpha $=.05$, the critical $t$-value was $2.00\left(f^{2}=.18 ; \mathrm{G}^{*}\right.$ Power; Erdfelder et al. 1996). Participants were recruited via the Prolific platform (www.prolific.co). Twenty-three participants were from the USA, 16 from the UK, 5 from Canada, 5 from Poland, 5 from Spain, 2 from Italy, and 1 each from Germany, Greece, Hungary, Ireland, and Portugal. Seventeen participants indicated that they had been diagnosed with psychiatric or psychological disorder (4 with depression, 2 with anxiety, 6 with depression and anxiety, 1 with bipolar disorder, 1 with posttraumatic stress disorder, 3 “other”).

Seven participants indicated that they personally knew at least one person who has been diagnosed with COVID-19, and one participant knew someone who died from COVID-19. Participants gave consent prior to participation and were compensated up to $\$ 10$. The study was approved by the local IRB. 


\section{Experimental Design, Measures, and Procedure}

The study was conducted on March 23, 2020. The design was correlational in nature, and participants completed all questionnaires via the Prolific platform (www.prolific.co). Questionnaires included the FFIS (Zabelina \& Condon, in press), the PROMIS (Cella et al., 2010), the Social and Emotional Loneliness Scale (Gierveld \& Van Tilburg, 2006), demographics, and other questionnaires as part of a larger study. All measures and procedures were identical to Study 1. Three participants answered the first attention check questions incorrectly, and none answered the second attention check question incorrectly.

\section{Results}

Replicating the results of Study 1, higher frequency of imagination was significantly associated with higher levels of anxiety during COVID-19 pandemic $(r=.44, p<.001)$, and with higher levels of loneliness $(r=.30, p=.019)$. In contrast to the results of Study 1 , higher frequency of imagination was also significantly associated with elevated anxiety before COVID-19 $(r=.27, p=.036)$. More positive imagination was linked with lower levels of anxiety both before $(r=-.66, p<.001)$ and during COVID-19 $(r=-.43, p=.001)$, and with lower levels of loneliness $(r=-.51, p<.001)$. In contract to Study 1 , age was not associated with any of the variables of interest (Table 4). 


\section{Table 4}

Pearson Correlation Coefficients for Imagination, Anxiety, Emotional and Social Loneliness, and Age in the International Community Sample.

\begin{tabular}{|c|c|c|c|c|c|c|c|c|}
\hline & 1 & 2 & 3 & 4 & 5 & 6 & 7 & 8 \\
\hline $\begin{array}{l}\text { 1. FFIS Frequency } \\
\text { 2. FFIS Complexity } \\
\text { 3. FFIS Emotional Valence } \\
\text { 4. FFIS Directedness } \\
\text { 5. Anxiety before COVID-19 } \\
\text { 6. Anxiety during COVID-19 } \\
\text { 7. Loneliness } \\
\text { 8. Age }\end{array}$ & -- & $\begin{array}{l}.28^{*} \\
--\end{array}$ & $\begin{array}{l}-.51^{* * *} \\
.21 \\
--\end{array}$ & $\begin{array}{l}.32^{*} \\
-.35^{* *} \\
.07 \\
--\end{array}$ & $\begin{array}{l}.27^{*} \\
-.19 \\
-.66^{* * *} \\
-. .08 \\
--\end{array}$ & $\begin{array}{l}.44 * * * \\
-.08 \\
-.43^{* *} \\
.06 \\
.36 * * \\
--\end{array}$ & $\begin{array}{l}.30^{*} \\
-.20 \\
-.51^{* * *} \\
-.10 \\
.38^{* *} \\
.44^{* * *} \\
--\end{array}$ & $\begin{array}{l}-.08 \\
.11 \\
.21 \\
.10 \\
-.22 \\
-.05 \\
.03 \\
--\end{array}$ \\
\hline $\begin{array}{l}\text { Mean } \\
\text { SD }\end{array}$ & $\begin{array}{l}3.50 \\
1.16\end{array}$ & $\begin{array}{l}4.40 \\
.88\end{array}$ & $\begin{array}{l}4.28 \\
1.17\end{array}$ & $\begin{array}{l}3.86 \\
.86\end{array}$ & $\begin{array}{l}1.63 \\
.64\end{array}$ & $\begin{array}{l}2.37 \\
.85\end{array}$ & $\begin{array}{l}2.76 \\
.80\end{array}$ & $\begin{array}{l}33.62 \\
13.78\end{array}$ \\
\hline
\end{tabular}

Note. $N=61 . \mathrm{FFIS}=$ Four Factor Imagination Scale. ${ }^{* * *} p<.001,{ }^{* *} p<.01,{ }^{*} p<.05$

There were no gender differences in imagination, anxiety, or emotional loneliness (Table

$5)$.

\section{Table 5}

Gender Differences in Imagination, Anxiety, and Loneliness in the International Community Sample.

\begin{tabular}{|c|c|c|c|c|c|c|c|}
\hline & \multicolumn{2}{|c|}{ Male } & \multicolumn{2}{|c|}{ Female } & \multirow[b]{2}{*}{$t$} & \multirow[b]{2}{*}{$p$} & \multirow[b]{2}{*}{$95 \% \mathrm{CI}$} \\
\hline & $M$ & $S D$ & $M$ & $S D$ & & & \\
\hline FFIS Frequency & 3.42 & .97 & 3.52 & 1.30 & .31 & .758 & {$[-.69 ; .50]$} \\
\hline FFIS Complexity & 4.50 & .91 & 4.28 & .87 & .96 & .343 & {$[-.24 ; .68]$} \\
\hline FFIS Emotional Valence & 4.36 & 1.03 & 4.24 & 1.30 & .41 & .687 & {$[-.49 ; .73]$} \\
\hline FFIS Directedness & 3.90 & .90 & 3.80 & .84 & .46 & .647 & {$[-.35 ; .55]$} \\
\hline Anxiety before COVID-19 & 1.57 & .61 & 1.68 & .68 & .65 & .520 & {$[-.44 ; .23]$} \\
\hline Anxiety during COVID-19 & 2.15 & .64 & 2.56 & .99 & 1.92 & .060 & {$[-.85 ; .02]$} \\
\hline Loneliness & 2.84 & .64 & 2.85 & .95 & .89 & .377 & {$[-.61 ; .23]$} \\
\hline
\end{tabular}

Note. $N=61$. FFIS $=$ Four Factor Imagination Scale. 
Separate multiple regression analyses were carried out to test the interactive effects between imagination (frequency, complexity, emotional valence, and directedness) and loneliness in predicting changes in anxiety during vs. before COVID-19. For the frequency of imagination, three predictors explained $8 \%$ of the variance in anxiety change, $\mathrm{R}^{2}=0.08, F(3,57)=2.78, p=$ .049. In line with results of Study 1, there was no main effect of imagination frequency, and no main effect of loneliness. Critically, replicating results of Study 1, there was a significant frequency of imagination x loneliness interaction (Table 6). Estimated means for the interaction were calculated for individuals low (-1 SD) versus high (1 SD) in the frequency of imagination and people low (-1 SD) versus high (1 SD) in loneliness, and are displayed in Figure 3. The interaction remained significant controlling for whether or not participants had been diagnosed with psychiatric or psychological disorders, $\beta=.27, t=2.17, p=.035$.

\section{Table 6}

Linear Multiple Regressions Predicting Anxiety Levels During vs. Before COVID-19 in the International Community Sample.

\begin{tabular}{lllllll}
\hline & $\mathrm{B}$ & $\mathrm{SE}$ & $\beta$ & $t$ & $p$ & $95 \% \mathrm{CI}$ \\
\hline DV: Anxiety levels during vs. before COVID-19 & & & & & \\
& & & & & & \\
FFIS Frequency & .20 & .11 & .23 & 1.75 & .086 & {$[-.03 ; .42]$} \\
Loneliness & .03 & .12 & .04 & .27 & .792 & {$[-.20 ; .26]$} \\
FFIS Freq x Loneliness & .23 & .11 & .26 & 2.09 & $.041^{*}$ & {$[.01 ; .45]$} \\
& & & & & & \\
\hline
\end{tabular}

Note. $N=61$. FFIS $=$ Four Factor Imagination Scale. ${ }^{*} p<.05$ 
Fig 3. Anxiety levels during vs. before COVID-19 as a function of frequency of imagination and loneliness in an international community sample. More positive values indicate more anxiety during compared to before COVID-19.

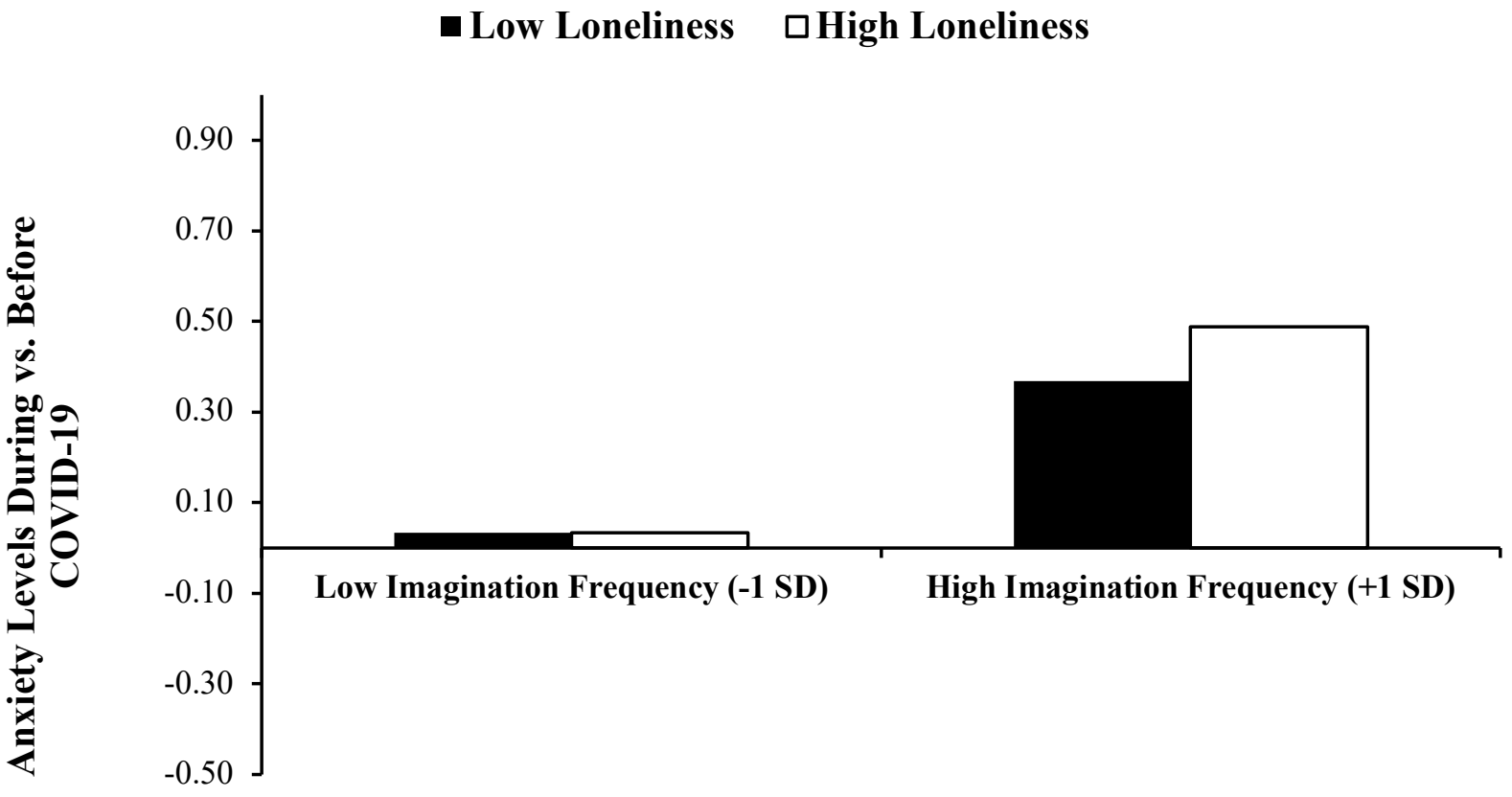

The main effects of complexity of imagination $(\beta=.10, t=.74, p=.464)$, loneliness $(\beta=$ $.14, t=1.01, p=.315)$, or their interaction $(\beta=.19, t=1.45, p=.153)$ in predicting the change in anxiety during vs. before COVID-19 were not statistically significant. There was also no main effect of the emotional valence of imagination $(\beta=.19, t=1.25, p=.217)$, or loneliness $(\beta=.25$, $t=1.65, p=.105)$, nor did they interact $(\beta=-.08, t=-.59, p=.560)$ in predicting changes in anxiety during vs. before COVID-19. Finally, there was no main effect of the directedness of imagination $(\beta=.13, t=1.03, p=.310)$, loneliness $(\beta=.16, t=1.22, p=.227)$, nor their interaction $(\beta$ $=.09, t=.67, p=.509)$ in predicting the change in anxiety during vs. before COVID-19.

\section{General Discussion}

In two studies we examined the association between imagination and anxiety, and the moderating effect of feelings of loneliness during the COVID-19 pandemic. Replicating in both 
studies, results showed that spending more time in one's imagination was linked with higher levels of anxiety during the pandemic. Anxiety before the pandemic was associated with frequency of imagination only in the international community sample, but not in the college sample. These findings are in line with previous studies conducted on large international community samples before the pandemic, which showed similar associations between frequency of imagination, elevated anxiety, and decreased psychological well-being (Zabelina \& Condon, in press). Thus, it may be that there is no association between imagination and anxiety in college population. Conversely, it may be that college students differ from the community sample in recalling their previous states of anxiety. Future studies will need to ask participants to report on their anxiety states before and during an adverse event to examine these questions further. In a related vein, COVID-19 as a major global trauma may be a limiting factor in the assessment of current and past anxiety symptoms and should be taken into consideration in the interpretation of the present findings.

Neuroimaging literature may provide insights into the potential mechanisms of the association between imagination and anxiety. Although little is known about the neural mechanisms of the frequency of imagination, mind-wandering - construct closely linked with imagination, was reported to activate regions of the default-mode network (Andrews-Hanna et al., 2010; Buckner et al., 2008; Christoff et al., 2009; Raichle et al., 2001). Both mind wandering and activity in the DMN have been linked to increased negative affect and lower psychological well-being (Mason et al., 2013; Zhao et al., 2007), and this association may be specific to unintentional, but not intentional mind wandering (Seli et al., 2019). The neural mechanisms of imagination frequency and anxiety should be examined in future work. 
Additionally, we found an interactive effect between frequency of imagination and loneliness in predicting the change in anxiety levels during, compared to before COVID-19. Specifically, we found that lonely people who spend more time imagining are more likely to also experience the highest elevation of anxiety during the pandemic. Previous work has shown that imagining significant others was associated with an alleviation of loneliness (Poerio et al., 2015; Poerio et al., 2016). Here we did not prompt participants to imagine nor did we ask about the content of their imagination. Instead, we report on the individual differences in trait imagination and its relation to the changes in anxiety as a function of feelings of loneliness.

Study 1 found that valence of imagination and loneliness interacted in such a way that the highest increase in anxiety during COVID-19 was in lonely people with more negative imagination. This finding was not replicated in Study 2, warranting further investigation. Complexity and directedness of imagination did not interact with loneliness in predicting changes in anxiety.

Finally, women reported higher anxiety both before and during COVID-19 pandemic in Study 1, in line with previous work (McLean et al., 2011), including a study in which African American women reported higher anxiety and depression symptoms compared to men (Chang, 2018). Here, we found no gender differences in anxiety in an international community sample in Study 2, suggesting that differences in anxiety may be more pronounced in college students.

Overall, in line with previous literature, our results show that at least some features of imagination - specifically its frequency, can have a dark side (Bacon \& Charlesford, 2018; Mar et al., 2012; Rhue \& Lynn, 1987). By specifying the link between imagination, anxiety, and loneliness, the present study makes an important contribution to the field and helps to understand the association between these constructs. Furthermore, this study is unique in that it was conducted 
during the unprecedented time of the COVID-19 pandemic, highlighting the association between these constructs during an arguably stressful time.

Our findings might inspire certain interventions, such as mindfulness training (Goldin \& Gross, 2010) for people who have a tendency to spend a lot of time in imaginative states and who also experience feelings of loneliness. Mindfulness interventions have been shown to be successful for reducing anxiety in a number of studies (Tacón et al., 2003). Mindful breathing has also been shown to reduce behavioral indicators of mind wandering (Mrazek et al., 2012). Therefore, mindfulness-based interventions may be especially useful for reducing the time one spends in certain imaginative states, and alleviating symptoms of anxiety.

\section{Limitations and Future Directions}

Because of the correlational nature of the present work, causal claims of the present findings are not substantiated. Whether too much time spent in one's imagination when lonely resulted in elevated anxiety levels, or if loneliness and anxiety increase people's imaginative states remains to be examines by future work. Data for the present studies were collected online using self-report measures. Future work may seek to replicate these findings with more objective measures of anxiety and imagination using larger sample sizes. Neuroimaging studies may yield important outcomes regarding neural mechanisms implicated in the frequency of imagination. Finally, although some participants reported clinically diagnosed psychological disorders, the majority of participants did not have a clinical diagnosis of anxiety disorder. Future studies may aim to investigate associations among imagination, anxiety, and loneliness in clinical populations. 


\section{Disclosure of Interest}

The authors have no conflict of interest. 


\section{References}

Andrews-Hanna, J. R., Reidler, J. S., Sepulcre, J., Poulin, R., \&Buckner, R. L. (2010). Functional-anatomic fractionation of the brain's default network, Neuron, 65(4), 550-562. https://doi.org/10.1016/j.neuron.2010.02.005.

Arcavi, A. (2003). The role of visual representations in the learning of mathematics. Educational Studies in Mathematics, 52(3), 215-241. https://doi.org/10.1023/A:1024312321077

Bacon, A. M., \& Charlesford, J. J. (2018). Investigating the association between fantasy proneness and emotional distress: the mediating role of cognitive coping strategies. Personality and Individual Differences, 135, 157-165. doi: 10.1016/j.paid.2018.07.003

Bai, Y., Lin, C-C., Lin, C-Y., Chen, J-Y., Chue, C-M., \& Chou, P. (2004). Survey of stress reactions among health care workers involved with the SARS outbreak. Psychiatric Services, 55(9), 1055-1057. doi: 10.1176/appi.ps.55.9.1055

Beaty, R. E., Chen, Q., Christensen, A. P., Qiu, J., Silvia, P. J., \& Schacter, D. L. (2018). Brain networks of the imaginative mind: Dynamic functional connectivity of default and cognitive control networks relates to openness to experience. Human Brain Mapping, 39(2), 811-821. https://doi.org/10.1002/hbm.23884

Brown, G. P., Macleod, A. K., Tata, P., \& Goddard, L. (2002). Worry and the simulation of future outcomes. Anxiety, Stress \& Coping, 15(1), 1-17. doi: 10.1080/10615800290007254

Buckner, R. L., Andrews-Hanna, J. R., \& Schacter, D. L. (2008). The brain's default network: anatomy, function, and relevance to disease. Annals of the New York Academy of Sciences, 1124, 1-38. doi: 10.1196/annals.1440.011

Cauberghe, V., Van Wesenbeeck, I., De Jans, S., Hudders, L., \& Ponnet, K. (in press). How adolescents use social media to cope with feelings of loneliness and anxiety during COVID- 
19 lockdown. Cyberpsychology, Behavior, and Social Networking. https://doi.org/10.1089/cyber.2020.0478

Cella, D., Riley, W., Stone, A., Rothrock, N., Reeve, B., Yount, S., Amtmann, D., Bode, R., Buysse, D., Choi, S., Cook, K., DeVellis, R., DeWalt, D., Fries, J. F., Gershon, R., Hahn, E. A., Lai, J-S., Pilkonis, P., Revicki, D., Rose, M., Weinfurt, K., \& Hays, R. (2010). Initial Adult Health Item Banks and First Wave Testing of the Patient-Reported Outcomes Measurement Information System (PROMISTM). Journal of Clinical Epidemiology, 63(11), 1179-1194. doi: 10.1016/j.jclinepi.2010.04.011

Centers for Disease and Control Prevention. (2020, June 10). Mental Health. https://www.cdc.gov/nchs/covid19/pulse/mental-health.htm

Chang, E. C. (2018). Relationship between loneliness and symptoms of anxiety and depression in African American men and women: Evidence for gender as a moderator. Personality and Individual Differences, 120, 138-143. https://doi.org/10.1016/j.paid.2017.08.035

Christoff, K., Gordon, A. M., Smallwood, J., Smith, R., \& Schooler, J. W. (2009). Experience sampling during fMRI reveals default network and executive system contributions to mind wandering. Proceedings of the National Academy of Sciences, 106(21), 8719-8724. doi: $10.1073 /$ pnas.0900234106

Erdfelder, E., Faul, F., \& Buchner, A. (1996). GPOWER: A general power analysis program. Behavior Research Methods, Instruments \& Computers, 28(1), 1-11. doi: 10.3758/BF03203630

Eres, R., Lim, M. H., Lanham, S., Jillard, C., \& Bates, G. (in press). Loneliness and emotion regulation: Implications of having social anxiety disorder. Australian Journal of Psychology. https://doi.org/10.1111/ajpy.12296 
Garry, M., \& Polaschek, D. L. (2000). Imagination and memory. Current Directions in Psychological Science, 9(1), 6-10. doi: 10.1111/1467-8721.00048

Gierveld, J. D. J., \& Tilburg, T. V. (2006). A 6-item scale for overall, emotional, and social loneliness: Confirmatory tests on survey data. Research on Aging, 28(5), 582-598. doi: $10.1177 / 0164027506289723$

Goldin, P. R., \& Gross, J. J. (2010). Effects of mindfulness-based stress reduction (MBSR) on emotion regulation in social anxiety disorder. Emotion, 10(1), 83. doi: 10.1037/a0018441

Guitard, T., Bouchard, S., Bélanger, C., \& Berthiaume, M. (2019). Exposure to a standardized catastrophic scenario in virtual reality or a personalized scenario in imagination for generalized anxiety disorder. Journal of Clinical Medicine, 8(3), 309. doi: 10.3390/jcm8030309

Hoffart, A., Johnson, S. U., \& Ebrahimi, O. V. (2020). Loneliness and social distancing during the COVID-19 pandemic: Risk factors and associations with psychopathology. Frontiers in Psychiatry, 11, 1297, https://doi.org/10.3389/fpsyt.2020.589127

Holmes, J. (2015). Attachment theory in clinical practice: A personal account. British Journal of Psychotherapy, 31(2), 208-228. https://doi.org/10.1111/bjp.12151

Huang, Y., \& Zhao, N. (2020). Generalized anxiety disorder, depressive symptoms and sleep quality during COVID-19 outbreak in China: a web-based cross-sectional survey. Psychiatry Research, 112954. doi: 10.1016/j.psychres.2020.112954

Jing, H. G., Madore, K. P., \& Schacter, D. L. (2016). Worrying about the future: An episodic specificity induction impacts problem solving, reappraisal, and well-being. Journal of Experimental Psychology: General, 145(4), 402. doi: 10.1037/xge0000142 
Killingsworth, M. A., \& Gilbert, D. T. (2010). A wandering mind is an unhappy mind. Science, 330(6006), 932-932. doi: 10.1126/science.1192439

Kroenke, K., Yu, Z., Wu, J., Kean, J., \& Monahan, P. O. (2014). Operating characteristics of PROMIS four-item depression and anxiety scales in primary care patients with chronic pain. Pain Medicine, 15(11), 1892-1901, https://doi.org/10.1111/pme.12537

LeBoutillier, N., \& Marks, D. F. (2003). Mental imagery and creativity: A meta-analytic review study. British Journal of Psychology, 94(1), 29-44. doi: 10.1348/000712603762842084

Mar, R. A., Mason, M. F., \& Livtack, A. (2012). How daydreaming related to life satisfaction, loneliness, and social support: The importance of gender and daydream content. Consciousness and Cognition, 21(1), 401-407. doi: 10.1016/j.concog.2011.08.001

Mason, M., Brown, K., Mar, R. A., \& Smallwood, J. (2013). Driver of discontent or escape vehicle: The affective consequences of mind wandering. Frontiers in Psychology, 4, 477. Doi: $10.3389 /$ fpsyg.2013.00477

McLean, C. P., Asnaani, A., Litz, B. T., \& Hofmann, S. G. (2011). Gender differences in anxiety disorders: prevalence, course of illness, comorbidity and burden of illness. Journal of Psychiatric Research, 45(8), 1027-1035.

Mrazek, M. D., Smallwood, J., \& Schooler, J. W. (2012). Mindfulness and mind-wandering: finding convergence through opposing constructs. Emotion, 12(3), 442-448.

Palgi, Y., Shrira, A., Ring, L., Bodner, E., Avidor, S., Bergman, Y., Cohen-Fridel, S., Keisari, S., \& Hoffman, Y. (2020). The loneliness pandemic: Loneliness and other concomitants of depression, anxiety and their comorbidity during the COVID-19 outbreak. Journal of Affective Disorders, 275, 109-111. https://doi.org/10.1016/j.jad.2020.06.036 
Peplau, L. A. (1982). Loneliness: A sourcebook of current theory, research, and therapy (Vol. 36). John Wiley \& Sons Inc.

Poerio, G. L., Totterdell, P., Emerson, L-M., \& Miles, E. (2015). Love is the triumph of the imagination: Daydreams abut significant others are associated with increased happiness, love and connection. Consciousness and Cognition, 33, 135-144. doi: 10.1016/j.con$\operatorname{cog} .2014 .12 .011$

Poerio, G. L., Totterdell, P., Emerson, L-M., \& Miles, E. (2016). Helping the heart grow fonder during absence: Daydreaming about significant others replenishes connectedness after induced loneliness. Cognition and Emotion, 30(6), 1197-1207. doi:

$10.1080 / 02699931.2015 .1049516$

Raichle M. E., MacLeod A. M., Snyder A. Z., Powers W. J., Gusnard D. A., \& Shulman G. L. (2001). A default mode of brain function. Proceedings of the National Academy of Sciences, 98, 676-82.

Rhue, J. W., \& Lynn, S. J. (1987). Fantasy proneness and psychopathology. Journal of Personality and Social Psychology, 53(2), 327-336. doi: 10.1037/0022-3514.53.2.327

Riley, S. N., \& Gabora, L. (2013). Evidence that threatening situations enhance creativity. arXiv preprint arXiv:1308.4245. https://ui.adsabs.harvard.edu/abs/2013arXiv1308.4245R

Sadoski, M., Goetz, E. T., Olivarez Jr, A., Lee, S., \& Roberts, N. M. (1990). Imagination in story reading: The role of imagery, verbal recall, story analysis, and processing levels. Journal of Reading Behavior, 22(1), 55-70. https://doi.org/10.1080/10862969009547694

Samli, A. C. (2011). From imagination to innovation: New product development for quality of life. Springer Science \& Business Media. 
Seli, P., Beaty, R. E., Marty-Dugas, J., \& Smilek, D. (2019). Depression, anxiety, and stress and the distinction between intentional and unintentional mind wandering. Psychology of Consciousness: Theory, Research, and Practice, 6(2), 163. doi: 10.1037/cns0000182

Schumaker, J. F., Shea, J. D., Monfries, M. M., \& Groth-Marnat, G. (1993). Loneliness and life satisfaction in Japan and Australia. The Journal of psychology, 127(1), 65-71. https://doi.org/10.1080/00223980.1993.9915543

Sprang, G., \& Silman, M. (2013). Posttraumatic Stress Disorder in Parents and Youth After Health-Related Disasters. Disaster Medicine and Public Health Preparedness, 7(1), 105110. doi: $10.1017 / \mathrm{dmp} .2013 .22$

Steptoe, A., Shankar, A., Demakakos, P., \& Wardle, J. (2013). Social isolation, loneliness, and all-cause mortality in older men and women. Proceedings of the National Academy of Sciences, 110(15), 5797-5801. https://doi.org/10.1073/pnas.1219686110

Stewart, E., Frank, H., Benito, K., Wellen, B., Herren, J., Skriner, L. C., \& Whiteside, S. P. (2016). Exposure therapy practices and mechanism endorsement: A survey of specialty clinicians. Professional Psychology: Research and Practice, 47(4), 303. doi: $10.1037 /$ pro0000094

Sündermann, O., Onwumere, J., Kane, F., Morgan, C., \& Kuipers, E. (2014). Social networks and support in first-episode psychosis: exploring the role of loneliness and anxiety. Social Psychiatry and Psychiatric Epidemiology, 49(3), 359-366.

Tacón, A. M., McComb, J., Caldera, Y., \& Randolph, P. (2003). Mindfulness meditation, anxiety reduction, and heart disease: a pilot study. Family \& Community Health, 26(1), 25-33. 
Taylor, M. R., Agho, K. E., Stevens, G. J., \& Raphael, B. (2008). Factors influencing psychological distress during a disease epidemic: Data from Australia's first outbreak of equine influenza. BMC Public Health, 8, 347. doi: 10.1186/1471-2458-8-347

Taylor, M., Carlson, S. M., Maring, B. L., Gerow, L., \& Charley, C. M. (2004). The characteristics and correlates of fantasy in school-age children: Imaginary companions, impersonation, and social understanding. Developmental Psychology, 40(6), 1173. DOI:

$10.1037 / 0012-1649.40 .6 .1173$

Taylor, S. E., Pham, L. B., Rivkin, I. D., \& Armor, D. A. (1998). Harnessing the imagination: Mental simulation, self-regulation, and coping. American Psychologist, 53(4), 429-439. doi: 10.1037//0003-066x.53.4.429

World Health Organization. (2020, April 29). Coronavirus disease (COVID-19) advice for the public. https://www.who.int/emergencies/diseases/novel-coronavirus-2019/advice-forpublic

Zabelina, D. L., \& Condon, D. M. (in press). The Four-Factor Imagination Scale (FFIS): A measure for assessing frequency, complexity, emotional valence, and directedness of imagination. Psychological Research. doi: 10.1007/s00426-019-01227-w

Zabelina, D. L. (in press). Imagination. In A.B. Bakker, F. Gander, W. Ruch, \& L. Tay (Eds.), Handbook in positive psychology assessment. Brussels, Belgium: European Association of Psychological Assessment (EAPA).

Zhao, X. H., Wang, P. J., Li, C. B., Hu, Z. H., Xi, Q., Wu, W. Y., \& Tang, X. W. (2007). Altered default mode network activity in patient with anxiety disorders: an fMRI study. European Journal of Radiology, 63(3), 373-378. https://doi.org/10.1016/j.ejrad.2007.02.006 
Zhou, H. X., Chen, X., Shen, Y. Q., Li, L., Chen, N. X., Zhu, Z. C., Castellanos, F. X., \& Yan, C. G. (2020). Rumination and the default mode network: Meta-analysis of brain imaging studies and implications for depression. Neuroimage, 206, 116287. https://doi.org/10.1016/j.neuroimage.2019.116287 